\title{
A Class of 1-Additive Sequences and Quadratic Recurrences
}

Julien Cassaigne and Steven R. Finch

\section{CONTENTS}

1. Introduction

2. Preliminaries

3. Ternary Quadratic Recurrence

4. Precisely Three Even Terms

5. Asymptotic Density

6. Questions

References

Electronic Availability
For odd $v \geq 5$, Schmerl and Spiegel have proved that the 1 -additive sequence $(2, v)$ has precisely two even terms and, consequently, is regular. For $5 \leq v \equiv 1 \bmod 4$, we prove, using a different approach, that the 1 -additive sequence $(4, v)$ has precisely three even terms. The proof draws upon the periodicity properties of a certain ternary quadratic recurrence.

Unlike the case of $(2, v)$, the regularity of $(4, v)$ can be captured by expressions in closed form. For example, its period can be written as an exponential sum of binary digit sums. Therefore the asymptotic density $\Delta(v)$ of $(4, v)$ tends to 0 as $v \rightarrow \infty$, but is misbehaved in the sense that

$$
\begin{aligned}
& \liminf _{\substack{v \rightarrow \infty \\
v \equiv 1 \bmod 4}}\left(\frac{v}{2}\right)^{2-\log _{2} 3} \Delta(v)=\frac{1}{4}, \\
& \underset{\substack{v \rightarrow \infty \\
v \equiv 1 \bmod 4}}{\limsup _{v}}\left(\frac{v}{2}\right)^{2-\log _{2} 3} \Delta(v)>0.27164 .
\end{aligned}
$$

This is proved using techniques adapted from Harborth and Stolarsky.

\section{INTRODUCTION}

Beginning with two positive integers $u<v$, Ulam [1964] defined the 1-additive sequence $(u, v)$ as

$$
(u, v)=a_{1}, a_{2}, a_{3}, \ldots
$$

where $a_{1}=u, a_{2}=v$ and $a_{n}$, for $n \geq 3$, is the least integer exceeding $a_{n-1}$ and possessing a unique representation of the form $a_{i}+a_{j}$, for $i<j$.

These sequences have been studied by, among others, Queneau [1972] and Finch [1991; 1992a; 1992b]. Many 1-additive sequences appear to behave quite erratically. For example, all the sequences $(1, v)$ for any $v>1$, as well as the sequence $(2,3)$, defy any simple characterization. 
In [Finch 1992a] it was conjectured that the sequence $(2, v)$, for odd $v \geq 5$, has precisely two even terms. Schmerl and Spiegel [1994] and, independently, Shirriff (private communication, 1993) have proved this conjecture. It then follows that $(2, v)$ is regular in the sense that the sequence of successive differences $a_{2}-a_{1}, a_{3}-a_{2}, \ldots$ is eventually periodic. As a corollary, $(2, v)$ has positive asymptotic density.

The following conjecture [Finch 1992b, Conj. 3] is based on empirical evidence obtained by computing several thousand terms of the relevant sequences directly according to the definition (see tables in [Finch 1992b]).

Conjecture 1.1. Assume $v \geq 5$ is odd.

(a) If $v \neq 2^{m}-1$ for any $m \geq 3$, the sequence $(4, v)$ has precisely three even terms: $4,2 v+4$, and $4 v+4$.

(b) When $v=2^{m}-1$ for some $m \geq 3$, the sequence $(4, v)$ has precisely four even terms: $4,2 v+4$, $4 v+4$, and $2\left(2 v^{2}+v-2\right)$.

A general proof of this conjecture, analogous to those of Schmerl and Spiegel or Shirriff, has not been found. However, we offer in Section 4 a proof in the case $v \equiv 1 \bmod 4$. This is possible only because of the periodicity properties of a certain ternary quadratic recurrence (Section 3 ). In Section 5 we derive a formula for the asymptotic density of $(4, v)$, for $v \equiv 1 \bmod 4$. Section 6 concludes with several related questions that remain open.

\section{PRELIMINARIES}

Assume that $v \geq 5$ is odd. Table 1 lists all terms of the sequence $(4, v)$ up to $4 v+8$, from which it follows that $(4, v)$ has at least three even terms. There are four cases, depending on the residue of $v$ modulo 8 . Notice, in particular, that the only even terms $\leq 4 v+8$ are $4,2 v+4$, and $4 v+4$.

For non-negative $h$ and $l$, let $z(h, l)$ be the binomial coefficient $\left(\begin{array}{c}h+l \\ l\end{array}\right)$ modulo 2 . For $h<0$ or $l<0$, let $z(h, l)=0$. Then:

Lemma 2.1. (a) For $h, l \geq 0$, we have $z(h, l)=1$ if and only if the bitwise "and" of the binary representations of $h$ and $l$ is zero [Long 1981].

(b) A recursive definition of $z$ is given by

$$
\begin{aligned}
z(2 i, 2 j) & =z(i, j), & z(2 i+1,2 j) & =z(i, j), \\
z(2 i, 2 j+1) & =z(i, j), & z(2 i+1,2 j+1) & =0,
\end{aligned}
$$

\begin{tabular}{|c|c|c|c|}
\hline $5 \bmod 8$ & $1 \bmod 8$ & $3 \bmod 8$ & $7 \bmod 8$ \\
\hline 4, & 4, & 4, & 4, \\
$\{v+4 j\}_{0}^{(v+3) / 4}$, & $\{v+4 j\}_{0}^{(v+3) / 4}$, & $\{v+4 j\}_{0}^{(v+1) / 4}$, & $\{v+4 j\}_{0}^{(v+1) / 4}$, \\
$2 v+4$, & $2 v+4$, & $2 v+4$, & $2 v+4$, \\
$\{2 v+7+4 j\}_{0}^{(v-5) / 4}$, & $\{2 v+7+4 j\}_{0}^{(v-5) / 4}$, & $\{2 v+5+4 j\}_{0}^{(v-3) / 4}$, & $\{2 v+5+4 j\}_{0}^{(v-3) / 4}$, \\
$\{3 v+4+8 j$, & $\{3 v+4+8 j$, & $\{3 v+4+8 j$, & $\{3 v+4+8 j$, \\
$3 v+6+8 j$, & $3 v+6+8 j$, & $3 v+6+8 j$, & $3 v+6+8 j$, \\
$3 v+10+8 j\}_{0}^{(v-13) / 8}$, & $3 v+10+8 j\}_{0}^{(v-9) / 8}$, & $3 v+10+8 j\}_{0}^{(v-11) / 8}$, & $3 v+10+8 j\}_{0}^{(v-7) / 8}$, \\
$4 v-1$, & $4 v+3$, & $4 v+1$, & $4 v+4$, \\
$4 v+1$, & $4 v+4$, & $4 v+3$, & $4 v+5$, \\
$4 v+4$, & $4 v+5$ & $4 v+4$, & \\
$4 v+5$, & & $4 v+7$ & \\
$4 v+7$ & & & \\
\hline
\end{tabular}

TABLE 1. The initial terms of the 1 -additive sequence $(4, v)$, for $v \geq 5$ odd, are determined by the residue of $v$ modulo 8. The terms are listed vertically to facilitate comparison among the four cases. The notation $\left\{f_{1}(j), \ldots, f_{n}(j)\right\}_{0}^{k}$ denotes the subsequence $f_{1}(0), \ldots, f_{n}(0), f_{1}(1), \ldots, f_{n}(1), \ldots, f_{1}(k), \ldots, f_{n}(k)$ when $k \geq 0$ and the empty subsequence when $k<0$. 
together with the initial conditions

$$
\begin{aligned}
z(0,0) & =1, \\
z(0,-1) & =z(-1,0)=z(-1,-1)=0 .
\end{aligned}
$$

(c) For a fixed $h$, the sequence $z(h, l)$ is periodic with (minimal) period $2^{g}$, where $g$ is the smallest integer such that $2^{g}>h$. Moreover

$$
\sum_{l=0}^{2^{g}-1} z(h, l)=2^{g-\#(h)}
$$

[Wolfram 1984], where \# $(h)$ denotes the number of ones in the binary expansion of $h$ and the summation is ordinary addition.

(d) Let $h^{0}, h^{1}, \ldots$ be the bits in the binary expansion of $h$, starting with the least significant bit, and likewise for $l^{0}, l^{1}, \ldots$ Define $i>0$ to be the smallest integer for which $\left(l^{i}, h^{i}\right) \neq\left(l^{0}, h^{0}\right)$. Then $z(h, l)=1$ implies

$$
z\left(h-h^{0}-h^{i}, l+l^{0}+l^{i}\right)=z\left(h+h^{0}+h^{i}, l-l^{0}-l^{i}\right)=1 .
$$

To prove this last statement, observe that by part (a) we cannot have $\left(l^{j}, h^{j}\right)=(1,1)$ for any $j$. If $l^{0}=l^{i}=0$ or $h^{0}=h^{i}=0$, the conclusion is immediate. By symmetry, it suffices to consider the case $\left(l^{0}, h^{0}, l^{i}, h^{i}\right)=(1,0,0,1)$. We have

$$
l=\cdots 0(1) 1 \quad \text { and } \quad h=\cdots 1(0) 0
$$

in binary, where the digit in parentheses is repeated $i-1$ times and where the beginning is unspecified. Then

$$
\begin{aligned}
h-h^{0}-h^{i} & =h-1=\cdots 0(1) 1 \\
l+l^{0}+l^{i} & =l+1=\cdots 1(0) 0 \\
h+h^{0}+h^{i} & =h+1=\cdots 1(0) 1 \\
l-l^{0}-l^{i} & =l-1=\cdots 0(1) 0,
\end{aligned}
$$

from which the desired formula follows.

\section{TERNARY QUADRATIC RECURRENCE}

Assume for the remainder of this paper that $v=$ $4 k+1$, where $k \geq 1$, and let $a_{1}, a_{2}, \ldots$ be the sequence $(4, v)$. Let $t$ satisfy $a_{t}=4 v+4$; one sees from Table 1 that $t=\frac{1}{8}(7 v+41)$ if $v \equiv 1 \bmod 8$ and $t=\frac{1}{8}(7 v+37)$ if $v \equiv 5 \bmod 8$. Define the infinite sequence

$$
\langle 4, v\rangle=b_{1}, b_{2}, b_{3}, \ldots
$$

where $b_{s}=a_{s}$ for $1 \leq s \leq t$ and $b_{n}$, for $n \geq t+1$, is the least odd integer exceeding $b_{n-1}$ and possessing a unique representation of the form $b_{i}+b_{j}$, for $i<j$. The claim that $(4, v)$ has precisely three even terms is equivalent to the claim that $(4, v)=\langle 4, v\rangle$. We shall associate to $\langle 4, v\rangle$ a certain ternary quadratic recurrence. Key properties of the recurrence will then give rise to a proof of the claim.

Define $x_{n}$, for $n>8 k+4$, as the number of representations of $2(n-6 k-4)+1$ as a sum $b_{i}+b_{j}$, with $i<j$. Since a sum of two integers is odd if and only if exactly one of the integers is even, it follows that

$x_{n}=\delta\left(x_{n-2}-1\right)+\delta\left(x_{n-4 k-3}-1\right)+\delta\left(x_{n-8 k-4}-1\right)$,

where $\delta(0)=1$ and $\delta(r)=0$ for $r \neq 0$. One way to simplify this formula is to let $x_{n}^{*}$ denote $x_{n} \bmod 3$; we obtain the ternary quadratic recurrence

$$
\begin{aligned}
x_{n}^{*}=2\left(x_{n-2}^{*}\left(x_{n-2}^{*}+1\right)+x_{n-4 k-3}^{*}\left(x_{n-4 k-3}^{*}+1\right)\right. \\
\left.+x_{n-8 k-4}^{*}\left(x_{n-8 k-4}^{*}+1\right)\right) \bmod 3
\end{aligned}
$$

with initial data

$$
\left(x_{1}^{*}, x_{2}^{*}, \ldots, x_{8 k+3}^{*}, x_{8 k+4}^{*}\right)=(0,0,, \ldots, 0,1) .
$$

The periods of $\left\{x_{n}^{*}\right\}$ and $\left\{x_{n}\right\}$ are clearly the same (since $\left\{x_{n}^{*}\right\}$ and $\left\{x_{n}\right\}$ can be expressed in terms of each other). There is, however, no known general treatment of quadratic recurrences available.

An alternative way to simplify (3.1) is to let $y_{n}=$ $\delta\left(x_{n}-1\right)$; then $y_{n}$ indicates mere membership in $\langle 4, v\rangle$. We obtain in this case the binary nonlinear recurrence

$$
y_{n}=\delta\left(y_{n-2}+y_{n-4 k-3}+y_{n-8 k-4}-1\right)
$$

with initial data

$$
\left(y_{1}, y_{2}, \ldots, y_{8 k+3}, y_{8 k+4}\right)=(0,0, \ldots, 0,1) .
$$


The periods of $\left\{y_{n}\right\}$ and $\left\{x_{n}\right\}$ are again clearly the same. It is remarkable that a closed form expression for $y_{n}$ exists, as we proceed to show.

Lemma 3.1. Write $n$ as $n=8(2 k+1) l+4 c+r$, with $l \geq-1,2 k+1 \leq c \leq 6 k+2$ and $1 \leq r \leq 4$. Then $y_{n}$, as defined by (3.2), is given by

0

$\begin{array}{ll}z(2 k+1, l) & \text { if } r=1 \text { and } c=5 k+3, \\ 0 & \text { if } r=1 \text { and } 5 k+4 \leq c \leq 6 k+2, \\ z(c-2 k-1, l) & \text { if } r=2 \text { and } 2 k+1 \leq c \leq 4 k+1, \\ 0 & \text { if } r=2 \text { and } 4 k+2 \leq c \leq 6 k+2, \\ 0 & \text { if } r=3 \text { and } 2 k+1 \leq c \leq 3 k, \\ z(c-3 k-1, l) & \text { if } r=3 \text { and } 3 k+1 \leq c \leq 5 k+2, \\ 0 & \text { if } r=3 \text { and } 5 k+3 \leq c \leq 6 k+2, \\ z(c-2 k, l) & \text { if } r=4 \text { and } 2 k+1 \leq c \leq 4 k, \\ 1-z(2 k+1, l) & \text { if } r=4 \text { and } c=4 k+1, \\ z(c-4 k-1, l) & \text { if } r=4 \text { and } 4 k+2 \leq c \leq 6 k+1, \\ 1-z(2 k+1, l) & \text { if } r=4 \text { and } c=6 k+2 .\end{array}$

Proof. By induction on $n$, using the relation

$$
z(h, l)=z(h-1, l)+z(h, l-1) \bmod 2 .
$$

If $1 \leq n \leq 8 k+4$, then $l=-1$ and $y_{n}$ is easily shown to be correct. The inductive step assumes that the claim is proved for values less than $n$, where $n \geq 8 k+5$. We will indicate the dependence of $(l, c, r)$ on such values, but not at $n$ itself. Table 2 exhibits the 25 cases with relevant parameter values. Using this, it is a straightforward (although tedious) matter to complete the proof. For example, if $r=3$ and $c=5 k+3$, we get

$x_{n}=y_{n-2}+y_{n-4 k-3}+y_{n-8 k-4}=2 z(1, l)+z(2 k+1, l)$.

If $l$ is even, then $z(1, l)=1$ and hence $y_{n}=0$; if $l$ is odd, then $z(1, l)=z(2 k+1, l)=0$ and again $y_{n}=0$. The other cases are similar.

The experimental origins of Table 2 are worth commenting on. The information we had at start was the conjectured period, $P=(2 k+1) 2^{m+3}$, of the sequence $\left\{y_{n}\right\}$, where $m$ is the smallest integer satisfying $2^{m}>2 k+1$. The fact that $P$ is a product is a hint of some additional structure. We arranged $\left\{y_{n}\right\}$, for small $k$, into rectangles of shape $a \times b$, with $a b=P$. The idea behind this was that a period like $a b$ can be the result of the superposition of two phenomena of period $a$ and $b$ (at least when the factors are relatively prime), and when one writes the sequence in rows of length $b$, any regularity in columns is a symptom of something of period $a$.

Here the most striking result was for $a=2^{m+1}$ and $b=4(2 k+1)$ : vertical, horizontal, and diagonal lines clearly appeared, for every value of $k$, reminiscent of Pascal triangle mod 2. For example, with $k=3$, we obtained

$$
\begin{aligned}
& 0000000000000000000000000001 \\
& 0101010101010111011101110110 \\
& 0011001100110011100100010000 \\
& 0100000101000011010000110101 \\
& 0010000100100001000000010001 \\
& 0101010000000011011101000001 \\
& 0011001000000001000100000001 \\
& 0100000000000011010000000001 \\
& 0010000000000001000000000001 \\
& 0101010101010110001000100011 \\
& 0001000100010000000000000001 \\
& 0100000101000010000000100001 \\
& 0000000100000000000000000001 \\
& 0101010000000010001000000001 \\
& 0001000000000000000000000001 \\
& 0100000000000010000000000001
\end{aligned}
$$

Some patterns, especially in the bottom right, indicated that something happens every second row and every fourth column. This suggested dividing the rectangle into four subrectangles of shape $2(2 k+1) \times 2^{m}$, according to $n \bmod 4$ :

$\begin{array}{lllll}00000000000000 & 00000001111111 & 00000000001111 & 00000011111110\end{array}$ $\begin{array}{llllll}00001000000000 & 00000001010101 & 11110000001010 & 11111100101011\end{array}$ $\begin{array}{lllll}00001000000000 & 00000001010101 & 11110000001010 & 11111100101011 \\ 00000000000000 & 00000001100110 & 10100000001100 & 01010111001101\end{array}$ $\begin{array}{llllll}00000000000000 & 00000001000100 & 11000000001000 & 10011010001001\end{array}$ $\begin{array}{llllll}00000000000000 & 00000001111000 & 10000000001111 & 00010011110001\end{array}$ $\begin{array}{lllll}00000000000000 & 00000001111000 & 10000000001111 & 00010011110001 \\ 00000000000000 & 00000001010000 & 00000000001010 & 11100010100001\end{array}$ $\begin{array}{lllll}00000000000000 & 00000001010000 & 00000000001010 & 11100010100001 \\ 00000000000000 & 00000001100000 & 00000000001100 & 01000011000001\end{array}$ $\begin{array}{llllll}00000000000000 & 00000001000000 & 00000000001000 & 10000010000001\end{array}$

The second and third subrectangles are clearly Pascal's triangle shifted by $2 k+1$ and $3 k+1$. The fourth is more complicated, but can be interpreted as two overlapping triangles (with addition mod 2 in the two overlap points), shifted $2 k$ and $4 k+1$. With these observations, one can easily guess the formulas of Lemma 3.1.

The expression for $y_{n}$ in Lemma 3.1 is fundamental to our analysis of the 1-additive sequence $(4,4 k+1)$. The values of $n$ for which $y_{n}$ is possibly nonzero are of special interest. Before continuing, we define ten sets of positive integers that depend on $(l, c, r)$ : 


$$
\begin{aligned}
A & =\{n: r=2 \text { and } 2 k+2 \leq c \leq 4 k+1\} \\
B & =\{n: r=3 \text { and } 3 k+2 \leq c \leq 5 k+1\} \\
C & =\{n: r=4 \text { and } 2 k+1 \leq c \leq 4 k\} \\
D & =\{n: r=4 \text { and } 4 k+2 \leq c \leq 6 k+1\} \\
E & =\{n: r=2 \text { and } c=2 k+1\} \\
F & =\{n: r=3 \text { and } c=3 k+1\} \\
G & =\{n: r=1 \text { and } c=5 k+3\} \\
H & =\{n: r=3 \text { and } c=5 k+2\} \\
I & =\{n: r=4 \text { and } c=4 k+1\} \\
J & =\{n: r=4 \text { and } c=6 k+2\}
\end{aligned}
$$

\begin{tabular}{|c|c|c|c|c|c|c|c|}
\hline$r$ & $c$ & $(l, c, r)_{n-2}$ & $(l, c, r)_{n-4 k-3}$ & $(l, c, r)_{n-8 k-4}$ & $y_{n-2}$ & $y_{n-4 k-3}$ & $y_{n-8 k-4}$ \\
\hline 1 & $c=2 k+1$ & $(l-1,6 k+2,3)$ & $(l-1,5 k+2,2)$ & $(l-1,4 k+2,1)$ & 0 & 0 & 0 \\
\hline 1 & $2 k+2 \leq c \leq 3 k+1$ & $(l, c-1,3)$ & $(l-1, c+3 k+1,2)$ & $(l-1, c+2 k+1,1)$ & 0 & 0 & 0 \\
\hline 1 & $c=3 k+2$ & $(l, 3 k+1,3)$ & $(l, 2 k+1,2)$ & $(l-1,5 k+3,1)$ & 1 & 1 & $z(2 k+1, l-1)$ \\
\hline 1 & $3 k+3 \leq c \leq 4 k+1$ & $(l, c-1,3)$ & $(l, c-k-1,2)$ & $(l-1, c+2 k+1,1)$ & $z(c-3 k-2, l)$ & $z(c-3 k-2, l)$ & 0 \\
\hline 1 & $4 k+2 \leq c \leq 5 k+2$ & $(l, c-1,3)$ & $(l, c-k-1,2)$ & $(l, c-2 k-1,1)$ & $z(c-3 k-2, l)$ & $z(c-3 k-2, l)$ & 0 \\
\hline 1 & $c=5 k+3$ & $(l, 5 k+2,3)$ & $(l, 4 k+2,2)$ & $(l, 3 k+2,1)$ & $z(2 k+1, l)$ & 0 & 0 \\
\hline 1 & $5 k+4 \leq c \leq 6 k+2$ & $(l, c-1,3)$ & $(l, c-k-1,2)$ & $(l, c-2 k-1,1)$ & 0 & 0 & 0 \\
\hline 2 & $c=2 k+1$ & $(l-1,6 k+2,4)$ & $(l-1,5 k+2,3)$ & $(l-1,4 k+2,2)$ & $1-z(2 k+1, l-1)$ & $z(2 k+1, l-1)$ & 0 \\
\hline 2 & $2 k+2 \leq c \leq 3 k+1$ & $(l, c-1,4)$ & $(l-1, c+3 k+1,3)$ & $(l-1, c+2 k+1,2)$ & $z(c-2 k-1, l)$ & 0 & 0 \\
\hline 2 & $3 k+2 \leq c \leq 4 k+1$ & $(l, c-1,4)$ & $(l, c-k-1,3)$ & $(l-1, c+2 k+1,2)$ & $z(c-2 k-1, l)$ & 0 & 0 \\
\hline 2 & $c=4 k+2$ & $(l, 4 k+1,4)$ & $(l, 3 k+1,3)$ & $(l, 2 k+1,2)$ & $1-z(2 k+1, l)$ & 1 & 1 \\
\hline 2 & $4 k+3 \leq c \leq 6 k+2$ & $(l, c-1,4)$ & $(l, c-k-1,3)$ & $(l, c-2 k-1,2)$ & $z(c-4 k-2, l)$ & $z(c-4 k-2, l)$ & $z(c-4 k-2, l)$ \\
\hline 3 & $2 k+1 \leq c \leq 3 k$ & $(l, c, 1)$ & $(l-1, c+3 k+1,4)$ & $(l-1, c+2 k+1,3)$ & 0 & $z(c-k, l-1)$ & $z(c-k, l-1)$ \\
\hline 3 & $c=3 k+1$ & $(l, 3 k+1,1)$ & $(l-1,6 k+2,4)$ & $(l-1,5 k+2,3)$ & 0 & $1-z(2 k+1, l-1)$ & $z(2 k+1, l-1)$ \\
\hline 3 & $3 k+2 \leq c \leq 4 k+1$ & $(l, c, 1)$ & $(l, c-k-1,4)$ & $(l-1, c+2 k+1,3)$ & 0 & $z(c-3 k-1, l)$ & 0 \\
\hline 3 & $4 k+2 \leq c \leq 5 k+1$ & $(l, c, 1)$ & $(l, c-k-1,4)$ & $(l, c-2 k-1,3)$ & 0 & $z(c-3 k-1, l)$ & 0 \\
\hline 3 & $c=5 k+2$ & $(l, 5 k+2,1)$ & $(l, 4 k+1,4)$ & $(l, 3 k+1,3)$ & 0 & $1-z(2 k+1, l)$ & 1 \\
\hline 3 & $c=5 k+3$ & $(l, 5 k+3,1)$ & $(l, 4 k+2,4)$ & $(l, 3 k+2,3)$ & $z(2 k+1, l)$ & $z(1, l)$ & $z(1, l)$ \\
\hline 3 & $5 k+4 \leq c \leq 6 k+2$ & $(l, c, 1)$ & $(l, c-k-1,4)$ & $(l, c-2 k-1,3)$ & 0 & $z(c-5 k-2, l)$ & $z(c-5 k-2, l)$ \\
\hline 4 & $c=2 k+1$ & $(l, 2 k+1,2)$ & $(l-1,5 k+3,1)$ & $(l-1,4 k+2,4)$ & 1 & $z(2 k+1, l-1)$ & $z(1, l-1)$ \\
\hline 4 & $2 k+2 \leq c \leq 3 k$ & $(l, c, 2)$ & $(l-1, c+3 k+2,1)$ & $(l-1, c+2 k+1,4)$ & $z(c-2 k-1, l)$ & 0 & $z(c-2 k, l-1)$ \\
\hline 4 & $3 k+1 \leq c \leq 4 k$ & $(l, c, 2)$ & $(l, c-k, 1)$ & $(l-1, c+2 k+1,4)$ & $z(c-2 k-1, l)$ & 0 & $z(c-2 k, l-1)$ \\
\hline 4 & $c=4 k+1$ & $(l, 4 k+1,2)$ & $(l, 3 k+1,1)$ & $(l-1,6 k+2,4)$ & $z(2 k, l)$ & 0 & $1-z(2 k+1, l-1)$ \\
\hline 4 & $4 k+2 \leq c \leq 6 k+1$ & $(l, c, 2)$ & $(l, c-k, 1)$ & $(l, c-2 k-1,4)$ & 0 & 0 & $z(c-4 k-1, l)$ \\
\hline 4 & $c=6 k+2$ & $(l, 6 k+2,2)$ & $(l, 5 k+2,1)$ & $(l, 4 k+1,4)$ & 0 & 0 & $1-z(2 k+1, l)$ \\
\hline
\end{tabular}

These sets, which we will use extensively in the next section, are disjoint and their union strictly contains $\left\{n: y_{n}=1\right\}$ (by Lemma 3.1). Note that $y_{n}$ takes the following forms over the various sets:

$$
\begin{aligned}
n \in A & \Rightarrow y_{n}=z(h, l) \quad \text { with } h=c-2 k-1 \\
n \in B & \Rightarrow y_{n}=z(h, l) \quad \text { with } h=c-3 k-1 \\
n \in C & \Rightarrow y_{n}=z(h, l) \quad \text { with } h=c-2 k \\
n \in D & \Rightarrow y_{n}=z(h, l) \quad \text { with } h=c-4 k-1 \\
n \in E & \Rightarrow y_{n}=1 \\
n \in F & \Rightarrow y_{n}=1 \\
n \in G & \Rightarrow y_{n}=z(2 k+1, l) \\
n \in H & \Rightarrow y_{n}=z(2 k+1, l) \\
n \in I & \Rightarrow y_{n}=1-(2 k+1, l) \\
n \in J & \Rightarrow y_{n}=1-(2 k+1, l)
\end{aligned}
$$

These sets are of two types: $A, B, C, D$ are intervals with a new parameter $h$, ranging in the interval $1 \leq h \leq 2 k$; while $E, F, G, H, I, J$ are isolated points.

TABLE 2. Parameter values for the proof of Lemma 3.1. 


\section{PRECISELY THREE EVEN TERMS}

Most of this section will be devoted to proving the following crucial fact.

Lemma 4.1. Suppose that $y_{p}=y_{q}=1$ with $p \neq q$, and that $p+q>20 k+13$. Then there exists $d \neq 0$ such that $y_{p+d}=y_{q-d}=1$ with $p+d \neq q-d$ and $p+d \neq q$.

Proof. Assume that $p \in X$ and $q \in Y$, and set $w=8(2 k+1)$. For every pair $(X, Y)$, we have to find an admissible value of $d$; there are 55 cases, not counting reversals, which are dealt with using the symmetry $(p, q, d) \rightarrow(q, p,-d)$. We illustrate the derivation of these admissible values by focusing on six cases, summarized in Table 3 . The remaining 49 cases can be subjected to a similar analysis (see also Electronic Availability at the end of this article).

Case 1: Isolated points mapped to isolated points. Suppose that $(X, Y)=(E, F)$. Then

$$
\begin{aligned}
& p=w l_{p}+4(2 k+1)+2, \\
& q=w l_{q}+4(3 k+1)+3 .
\end{aligned}
$$

\begin{tabular}{|c|c|c|c|c|c|}
\hline$(X, Y)$ & $d$ & $\left(X^{\prime}, Y^{\prime}\right)$ & $\left(l_{p+d}, l_{q-d}\right)$ & $\left(h_{p+d}, h_{q-d}\right)$ & condition(s) \\
\hline \multirow[t]{2}{*}{$(E, F)$} & $w$ & $(E, F)$ & $\left(l_{p}+1, l_{q}-1\right)$ & & \multirow{2}{*}{ (NB: $l_{p}>0$ or $l_{q}>0$ ) } \\
\hline & $-w$ & $(E, F)$ & $\left(l_{p}-1, l_{q}+1\right)$ & & \\
\hline \multirow[t]{3}{*}{$(H, H)$} & $12 k+1$ & $(C, A)$ & $\left(l_{p}+1, l_{q}\right)$ & $(2 k, 1)$ & \\
\hline & $12 k+3$ & $(A, C)$ & $\left(l_{p}+1, l_{q}\right)$ & $(2 k, 1)$ & \\
\hline & $12 k+5$ & $(I, E)$ & $\left(l_{p}+1, l_{q}\right)$ & & \\
\hline \multirow[t]{2}{*}{$(C, E)$} & $4 k+3$ & $(B, H)$ & $\left(l_{p}, l_{q}-1\right)$ & $\left(h_{p},{ }_{-}\right)$ & $z\left(2 k+1, l_{q}-1\right)=1$ \\
\hline & 2 & $(A, J)$ & $\left(l_{p}, l_{q}-1\right)$ & $\left(h_{p},-\right)$ & $z\left(2 k+1, l_{q}-1\right)=0$ \\
\hline \multirow[t]{4}{*}{$(A, C)$} & $w l_{q}-4 h_{p}$ & $(E, C)$ & $\left(l_{p}+l_{q}, 0\right)$ & $\left(-, h_{p}+h_{q}\right)$ & $h_{p}+h_{q} \leq 2 k$ \\
\hline & $w l_{q}-4 h_{p}$ & $(E, D)$ & $\left(l_{p}+l_{q}, 0\right)$ & $\left(-, h_{p}+h_{q}-2 k-1\right)$ & $h_{p}+h_{q}>2 k+1$ \\
\hline & -2 & $(C, A)$ & $\left(l_{p}, l_{q}\right)$ & $\left(h_{p}, h_{q}\right)$ & $l_{p} \neq l_{q}$ \\
\hline & $4 h_{q}-4 h_{p}$ & $(A, C)$ & $\left(l_{p}, l_{q}\right)$ & $\left(h_{q}, h_{p}\right)$ & $l_{p}=l_{q}, h_{p} \neq h_{q}$ \\
\hline \multirow[t]{9}{*}{$(A, B)$} & $4 k+1$ & $(B, A)$ & $\left(l_{p}, l_{q}\right)$ & $\left(h_{p}, h_{q}\right)$ & $l_{p} \neq l_{q}$ \\
\hline & $4 h_{q}-4 h_{p}$ & $(A, B)$ & $\left(l_{p}, l_{q}\right)$ & $\left(h_{q}, h_{p}\right)$ & $l_{p}=l_{q}, h_{p} \neq h_{q}$ \\
\hline & $w$ & $(A, B)$ & $\left(l_{p}+1, l_{p}-1\right)$ & $\left(h_{p}, h_{p}\right)$ & $l_{p}=l_{q}, h_{p}=h_{q}, h^{0}+h^{i}=0, l^{0}+l^{i}=1 \quad\left(\mathrm{NB}: l_{p}>0\right)$ \\
\hline & -4 & $(E, B)$ & $\left(l_{p}, l_{p}\right)$ & $(-, 2)$ & $l_{p}=l_{q}, h_{p}=h_{q}=1, h^{0}+h^{i}=1, l^{0}+l^{i}=0$ \\
\hline & -4 & $(A, B)$ & $\left(l_{p}, l_{p}\right)$ & $\left(h_{p}-1, h_{p}+1\right)$ & $l_{p}=l_{q}, 1<h_{p}=h_{q}<2 k, h^{0}+h^{i}=1, l^{0}+l^{i}=0$ \\
\hline & -4 & $(A, H)$ & $\left(l_{p}, l_{p}\right)$ & $\left(2 k-1,{ }_{-}\right)$ & $l_{p}=l_{q}, h_{p}=h_{q}=2 k, h^{0}+h^{i}=1, l^{0}+l^{i}=0$ \\
\hline & $w-4$ & $(E, B)$ & $\left(l_{p}+1, l_{p}-1\right)$ & $(-, 2)$ & $l_{p}=l_{q}, h_{p}=h_{q}=1, h^{0}+h^{i}=1, l^{0}+l^{i}=1 \quad\left(\mathrm{NB}: l_{p}>0\right)$ \\
\hline & $w-4$ & $(A, B)$ & $\left(l_{p}+1, l_{p}-1\right)$ & $\left(h_{p}-1, h_{p}+1\right)$ & $l_{p}=l_{q}, 1<h_{p}=h_{q}<2 k, h^{0}+h^{i}=1, l^{0}+l^{i}=1 \quad\left(\mathrm{NB}: l_{p}>0\right)$ \\
\hline & $w-4$ & $(A, H)$ & $\left(l_{p}+1, l_{p}-1\right)$ & $\left(2 k-1,{ }_{-}\right)$ & $l_{p}=l_{q}, h_{p}=h_{q}=2 k, h^{0}+h^{i}=1, l^{0}+l^{i}=1 \quad\left(\mathrm{NB}: l_{p}>0\right)$ \\
\hline \multirow[t]{3}{*}{$(C, I)$} & 2 & $(A, A)$ & $\left(l_{p}, l_{q}\right)$ & $\left(h_{p}, 2 k\right)$ & $z\left(2 k+1, l_{q}-1\right)=1$ and $l_{p} \neq l_{q}$ \\
\hline & $8 k+4$ & $(D, J)$ & $\left(l_{p}, l_{q}-1\right)$ & $\left(h_{p},{ }_{-}\right)$ & $z\left(2 k+1, l_{q}-1\right)=0$ \\
\hline & $-8 k-4$ & $(D, J)$ & $\left(l_{p}-1, l_{q}\right)$ & $\left(h_{p},,_{-}\right)$ & $l_{p}$ odd (NB: true if $z\left(2 k+1, l_{q}-1\right)=1$ and $\left.l_{p}=l_{q}\right)$ \\
\hline
\end{tabular}

TABLE 3. Admissible values of $d$ for representative pairs $(X, Y)$ in the proof of Lemma 4.1 and the discussion following Theorem 4.2. We indicate the new sets $\left(X^{\prime}, Y^{\prime}\right)$ for which $p+d \in X^{\prime}$ and $q-d \in Y^{\prime}$, and the corresponding new values for $l$ and $h$. If there are several lines without conditions for a given pair $(X, Y)$, each of them is a solution. If there are several lines with conditions, at least one of the conditions is true for each relevant value of $(p, q)$. 
Choose $s \neq 0$ such that $-l_{p} \leq s \leq l_{q}$. This is always possible, except when $l_{p}=l_{q}=0$, in which case $p+q=20 k+13$, which is excluded. (In Table 3, we have taken $s= \pm 1$.) Let $d=s w$. Then

$$
\begin{aligned}
& p+d=w\left(l_{p}+s\right)+4(2 k+1)+2>0, \\
& q-d=w\left(l_{q}-s\right)+4(3 k+1)+3>0,
\end{aligned}
$$

and $p+d \in E=X^{\prime}, q-d \in F=Y^{\prime}$, hence $y_{p+d}=y_{q-d}=1$. This confirms the parameter values appearing on the first two lines of Table 3. Finally, $p+d \neq q-d$ since $X^{\prime} \neq Y^{\prime}$, and $p+d \neq q$ since $X^{\prime} \neq Y$. (This final step is often trivial, so we omit it from now on except in Case 4.)

Case 2: Isolated points mapped to interval endpoints. Suppose that $(X, Y)=(H, H)$. Then

$$
\begin{array}{ll}
p=w l_{p}+4(5 k+2)+3, & y_{p}=z\left(2 k+1, l_{p}\right), \\
q=w l_{q}+4(5 k+2)+3, & y_{q}=z\left(2 k+1, l_{q}\right) .
\end{array}
$$

Since $y_{p}=y_{q}=1$, we know that $z\left(2 k+1, l_{p}\right)=$ $z\left(2 k+1, l_{q}\right)=1$ and hence $l_{p}$ and $l_{q}$ are necessarily even. Let $d=12 k+1$. Then

$$
\begin{aligned}
& p+d=w\left(l_{p}+1\right)+4(4 k)+4, \\
& q-d=w l_{q}+4(2 k+2)+2 .
\end{aligned}
$$

We see that $p+d \in C, l_{p+d}=l_{p}+1, h_{p+d}=2 k$ and $q-d \in A, l_{q-d}=l_{q}, h_{q-d}=1$. To prove that $d=12 k+1$ is admissible, note that

$$
\begin{aligned}
y_{p+d} & =z\left(h_{p+d}, l_{p+d}\right) \\
& =z\left(2 k, l_{p}+1\right)=z\left(2 k+1, l_{p}\right)=y_{p}=1
\end{aligned}
$$

since $l_{p}$ is even and by Lemma 2.1(b). Note also that

$$
y_{q-d}=z\left(h_{q-d}, l_{q-d}\right)=z\left(1, l_{q}\right)=1
$$

because $l_{q}$ is even. (Two other admissible values for $\mathrm{d}$ are also given in Table 3.)

Case 3: Mixed cases mapped to mixed cases. Suppose that $(X, Y)=(C, E)$. Then

$$
\begin{aligned}
& p=w l_{p}+4\left(2 k+h_{p}\right)+4, \\
& q=w l_{q}+4(2 k+1)+2 .
\end{aligned}
$$

There are two subcases. First assume that

$$
z\left(2 k+1, l_{q}-1\right)=1 .
$$

Let $d=4 k+3$; then

$$
\begin{aligned}
p+d & =w l_{p}+4\left(3 k+1+h_{p}\right)+3, \\
q-d & =w\left(l_{q}-1\right)+4(5 k+2)+3 .
\end{aligned}
$$

We see that $p+d \in B, l_{p+d}=l_{p}, h_{p+d}=h_{p}$ and $q-d \in H, l_{q-d}=l_{q}-1$. Therefore

$$
\begin{aligned}
& y_{p+d}=z\left(h_{p}, l_{p}\right)=y_{p}=1, \\
& y_{q-d}=z\left(2 k+1, l_{q}-1\right)=1
\end{aligned}
$$

by assumption.

In the second subcase, assume instead that

$$
z\left(2 k+1, l_{q}-1\right)=0 .
$$

Let $d=2$. The analysis is similar to the first subcase.

Case 4: Intervals mapped to intervals (first example). Suppose that $(X, Y)=(A, C)$. Then

$$
\begin{aligned}
& p=w l_{p}+4\left(2 k+1+h_{p}\right)+2, \\
& q=w l_{q}+4\left(2 k+h_{q}\right)+4 .
\end{aligned}
$$

Again, there are two subcases. First assume that $h_{p}+h_{q} \neq 2 k+1$. Let $d=w l_{q}-4 h_{p}$; then

$$
\begin{aligned}
& p+d=w\left(l_{p}+l_{q}\right)+4(2 k+1)+2, \\
& q-d=4\left(2 k+h_{p}+h_{q}\right)+4 .
\end{aligned}
$$

We see that $p+d \in E$, hence $y_{p+d}=1$. If we have $2 \leq h_{p}+h_{q} \leq 2 k$, then $q-d \in C, l_{q-d}=0$ and $h_{q-d}=h_{p}+h_{q}$, hence $y_{q-d}=z\left(h_{p}+h_{q}, 0\right)=1$. If $2 k+2 \leq h_{p}+h_{q} \leq 4 k$, then $q-d \in D, l_{q-d}=0$ and $h_{q-d}=h_{p}+h_{q}-2 k-1$, hence

$$
y_{q-d}=z\left(h_{p}+h_{q}-2 k-1,0\right)=1 .
$$

In the second subcase, assume that $l_{p} \neq l_{q}$ or $h_{p} \neq h_{q}$. (Thus the two subcases are not disjoint, but they certainly exhaust all possibilities, since $h_{p}=h_{q}$ implies that $h_{p}+h_{q}$ cannot be odd).

If $l_{p} \neq l_{q}$, let $d=-2$. Then $p+d \in C, l_{p+d}=l_{p}$, $h_{p+d}=h_{p}$, hence $y_{p+d}=y_{p}=1$; also $q-d \in A$, 
$l_{q-d}=l_{q}, h_{q-d}=h_{q}$, hence $y_{q-d}=y_{q}=1$. Here $X^{\prime}=Y$, so we need $l_{p} \neq l_{q}$ to ensure $p+d \neq q$.

If $l_{p}=l_{q}$ and $h_{p} \neq h_{q}$, let $d=4\left(h_{q}-h_{p}\right)$. Then $p+d \in A, l_{p+d}=l_{p}=l_{q}, h_{p+d}=h_{q}$, hence $y_{p+d}=$ $y_{q}=1$; also $q-d \in C, l_{q-d}=l_{q}=l_{p}, h_{q-d}=h_{p}$, hence $y_{q-d}=y_{p}=1$. Here $X^{\prime}=X$, so we need $h_{p} \neq h_{q}$ to ensure $d \neq 0$.

Case 5: Intervals mapped to intervals (second example). Suppose that $(X, Y)=(A, B)$. Then

$$
\begin{aligned}
& p=w l_{p}+4\left(2 k+1+h_{p}\right)+2, \\
& q=w l_{q}+4\left(3 k+1+h_{q}\right)+3 .
\end{aligned}
$$

If $l_{p} \neq l_{q}$ or $h_{p} \neq h_{q}$, the procedure is similar to that for the second subcase of Case 4 . Thus assume that $l_{p}=l_{q}=l$ and $h_{p}=h_{q}=h$. Let $d=w\left(l^{0}+l^{i}\right)$ $-4\left(h^{0}+h^{i}\right)$, where we now utilize Lemma 2.1(d). (This definition gives rise to $d=w, d=-4$ and $d=w-4$ in Table 3.) Then

$$
\begin{aligned}
& p+d=w\left(l+l^{0}+l^{i}\right)+4\left(2 k+1+h-h^{0}-h^{i}\right)+2, \\
& q-d=w\left(l-l^{0}-l^{i}\right)+4\left(3 k+1+h+h^{0}+h^{i}\right)+3 .
\end{aligned}
$$

We have $l_{p+d}=l+l^{0}+l^{i}$ and

$$
\begin{cases}p+d \in A \text { and } h_{p+d}=h-h^{0}-h^{i} & \text { if } h>1, \\ p+d \in E & \text { if } h=1\end{cases}
$$

in either case, $y_{p+d}=z\left(h-h^{0}-h^{i}, l+l^{0}+l^{i}\right)=1$. We also have $l_{q-d}=l-l^{0}-l^{i} \geq 0$ and

$$
\begin{cases}q-d \in B, h_{q-d}=h+h^{0}+h^{i} & \text { if } h<2 k \text { or } h^{i}=0, \\ q-d \in H & \text { if } h=2 k \text { and } h^{i}=1\end{cases}
$$

in either case, $y_{q-d}=z\left(h+h^{0}+h^{i}, l-l^{0}-l^{i}\right)=1$.

This completes the sketch of the proof of the lemma. The last pair $(C, I)$ in Table 3 is discussed below.
Theorem 4.2. For any $k \geq 1$, the 1-additive sequence $(4,4 k+1)$ has precisely three even terms. Equivalently, $(4,4 k+1)=\langle 4,4 k+1\rangle$.

Proof. Set $v=4 k+1$, and suppose $(4, v)$ has an even term exceeding $4 v+4$. Let $e$ be the least such even term. Observe that $e$ is the first point of disagreement between $(4, v)$ and $\langle 4, v\rangle$, so $y_{n}$ indicates membership in $(4, v)$ for $n<\frac{1}{2} e+6 k+4$. Since $4+(4 v+4)=(v+4)+(3 v+4)$ and $(2 v+4)+$ $(4 v+4)=(3 v+2)+(3 v+6)$, we may rule out the possibility that $e$ is a sum of two even terms of $(4, v)$. Hence $e=f+g$, where $f$ and $g$ are odd terms. Setting

$$
p=\frac{1}{2}(f-1)+6 k+4, \quad q=\frac{1}{2}(g-1)+6 k+4,
$$

we have $y_{p}=y_{q}=1$ and

$$
\begin{aligned}
p+q & =\frac{1}{2} e+12 k+7 \\
& >\frac{1}{2}(4 v+8)+12 k+7=20 k+13 .
\end{aligned}
$$

The last inequality follows from Table 1.

By Lemma 4.1, there exists $d \neq 0$ for which $y_{p+d}=y_{q-d}=1$ with $p+d \neq q-d$ and $p+d \neq q$. Then

$e=(2(p+d-6 k-4)+1)+(2(q-d-6 k-4)+1)$,

which is a contradiction since $e=f+g$ was the unique representation of $e$ as a sum of two odd terms $f<g$.

Again, the experimental origins of Lemma 4.1 are worth mentioning. We used a model with strips of paper in order to find candidates for $d$ (modulo $w)$. Most of the cases can be solved with values of $d$ such that $(p, q) \in(X, Y)$ implies $(p+d, q-d) \in$ $\left(X^{\prime}, Y^{\prime}\right)$, with intervals being mapped to intervals, and isolated points to isolated points or interval

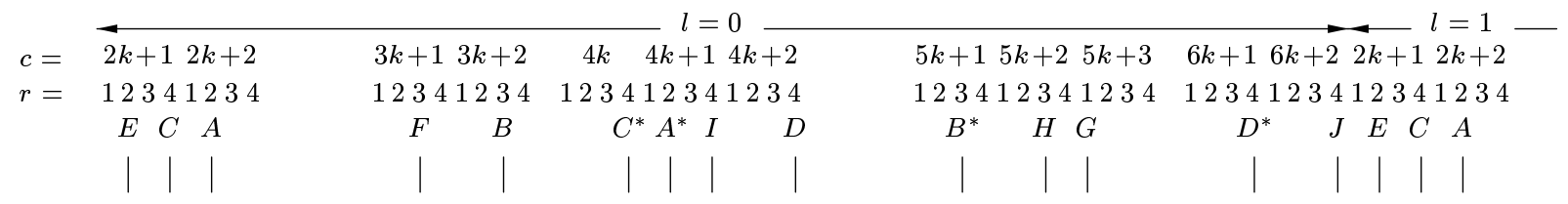

FIGURE 1. The proof of Lemma 4.1 was helped by the use of strips marked with ticks and letters $A-J$ in appropriate positions. 
endpoints. These conditions drastically reduce the number of candidates.

To construct the model, choose a (not necessarily integer) value of $k \geq 3$, and take two strips of ruled paper at least $32 k+16$ units long (one might take a unit to be around $2 \mathrm{~mm}$, with $k=3.25)$. The strips are graduated from $(l, c, r)=(0,2 k+1,1)$ to $(1,6 k+2,4)$. Along the edge of each strip, mark in three colors the positions of the isolated points $E$ $J$, the interval starting points $A-D$ and the interval ending points $A^{*}-D^{*}$ (each point appears twice, for $l=0$ and $l=1$ ). Figure 1 shows one such strip.

To use the model, place one strip against the other, so that point $X$ on strip 1 matches point $Y$ on strip 2, and so that their overlap encompasses more than half of their length. Consider the case $(X, Y)=(C, I)$, shown in Figure 2. Matches of markings indicate candidates for $\left(X^{\prime}, Y^{\prime}\right)$, in this case $(C, I),\left(A, A^{*}\right),\left(A^{*}, A\right),(I, C),(D, J),(H, G)$, $(G, H),(J, D)$. For the last four, the sum of l's has to decrease by 1 . Since $X$ is an interval, $X^{\prime}$ should be one too. Thus three cases are left: $(C, I)$, $\left(A, A^{*}\right)$ and $(D, J)$. Of these, mapping $(C, I)$ to $(C, I)$ would involve changing $l$; this is not easily done for intervals since values of $z(h, l)$ become perturbed. Mapping to $\left(A, A^{*}\right)$ and $(D, J)$ may or may not work: a few computations give the three conditions listed in Table 3. (Sometimes some more thought is needed to find the right variation of $l$ ).

This method does not exhaust all the cases, but only 11 out of 55 pairs $(X, Y)$ are left for which it is necessary to vary $h$ (see cases $3-5$ in the proof of Lemma 4.1).

\section{ASYMPTOTIC DENSITY}

Theorem 4.2 allows statements about the sequence $\left\{y_{n}\right\}$ to carry over to statements about the sequence $(4, v)$, where $v=4 k+1$ with $k \geq 1$. We exploit this connection here, obtaining formulas for the fundamental difference and the period of $(4, v)$.

Lemma 5.1. The sequence $\left\{y_{n}\right\}$ is periodic with minimal period $P=2^{m+3}(2 k+1)$, where $m$ is the smallest integer satisfying $2^{m}>2 k+1$.

Proof. We have expressed $\left\{y_{n}\right\}$ as a function of several $z(h, l)$, where $h \leq 2 k+1$. Hence it is clear by Lemma 2.1 (c) that $y_{n+P}=y_{n}$ for all $n$. To show that the period $p$ of $\left\{y_{n}\right\}$ is indeed $P$ (and not one of its divisors), observe first that $0=y_{p+1}=y_{p+2}=$ $\cdots=y_{p+8 k+3}$. By Lemma 3.1, we know that

$$
\begin{aligned}
y_{w l+8 k+6} & =y_{w l+4(2 k+1)+2}=1, \\
y_{w l+12 k+7} & =y_{w l+4(3 k+1)+3}=1
\end{aligned}
$$

for all $l$, that

$$
y_{w l+16 k+2}=y_{w l+4(4 k+2)+4}=z(1, l)=1
$$

if $l$ is even, and that

$$
\begin{aligned}
y_{w l+16 k+8} & =y_{w l+4(4 k+1)+4}=1-z(2 k+1, l)=1, \\
y_{w l+24 k+12} & =y_{w l+4(6 k+2)+4}=1-z(2 k+1, l)=1
\end{aligned}
$$

if $l$ is odd. This means that $p$ cannot be in the following intervals:

$$
\begin{array}{ll}
{[w l+3, w l+8 k+5]} & \text { for all } l, \\
{[w l+4 k+4, w l+12 k+6]} & \text { for all } l, \\
{[w l+8 k+9, w l+16 k+11]} & \text { if } l \text { is even, } \\
{[w l+8 k+5, w l+16 k+7]} & \text { if } l \text { is odd } \\
{[w l+16 k+9, w l+24 k+11]} & \text { if } l \text { is odd. }
\end{array}
$$

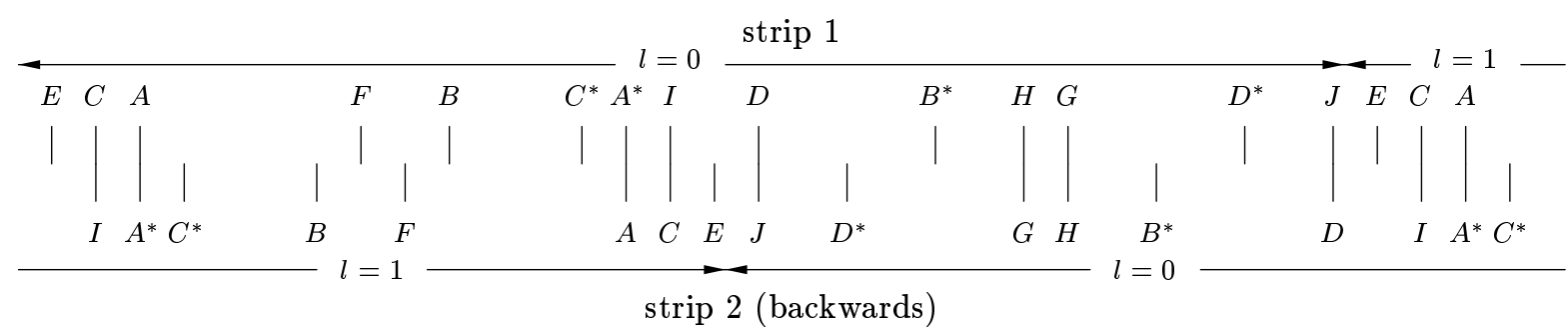

FIGURE 2. Juxtaposing two strips so that point $X$ on strip 1 matches point $Y$ on strip 2 shows the possible candidates for $\left(X^{\prime}, Y^{\prime}\right)$. Here $(X, Y)=(C, I)$. 
These intervals overlap, covering all positive integers except the points $w l+16 k+8$ when $l$ is odd. Hence the only candidates for $p$ must be of the form $w(l+1)$, for $l$ odd. Since $p$ divides $P=2^{m} w$, it is clear that $l+1=2^{j}$ for some $j \leq m$. Note that $j=m-1$ is impossible because

$$
y_{20 k+13}=y_{w 0+4(5 k+3)+1}=z(2 k+1,0)=1
$$

and

$$
y_{20 k+13+p}=y_{w(l+1)+4(5 k+3)+1}=z\left(2 k+1,2^{m-1}\right)=0
$$

$\left(\right.$ since $\left.2^{m-1}<2 k+1<2^{m}\right)$. Therefore $p=P$.

Lemma 5.2. The number of ones in a (minimal) period of the sequence $\left\{y_{n}\right\}$ is

$$
2^{m+2} \sum_{j=0}^{2 k} 2^{-\#(j)}
$$

where $m$ is the smallest integer satisfying $2^{m}>$ $2 k+1$.

Proof. By Lemma 2.1(c), we can write

$$
\begin{aligned}
\sum_{n=1}^{P} y_{n}= & \sum_{l=0}^{2^{m}-1} \sum_{r=1}^{4} \sum_{c=2 k+1}^{6 k+2} y_{w l+4 c+r} \\
= & \sum_{l=0}^{2^{m}-1}\left(z(2 k+1, l)+\sum_{j=0}^{2 k} z(j, l)\right. \\
& +\sum_{j=0}^{2 k+1} z(j, l)+\sum_{j=1}^{2 k} z(j, l)+1-z(2 k+1, l) \\
& \left.+\sum_{j=1}^{2 k} z(j, l)+1-z(2 k+1, l)\right) \\
= & 4 \sum_{l=0}^{2^{m}-1} \sum_{j=0}^{2 k} z(j, l)=4 \sum_{j=0}^{2 k} \sum_{l=0}^{2^{m}-1} z(j, l) \\
= & 2^{m+2} \sum_{j=0}^{2 k} 2^{-\#(j)} .
\end{aligned}
$$

Definition. Let $(u, v)=a_{1}, a_{2}, a_{3}, \ldots$ be a regular 1-additive sequence. The smallest positive integer $N$ such that $a_{N+n+1}-a_{N}=a_{n+1}-a_{n}$ for all sufficiently large $n$ is called the period. The value
$D=a_{N+n}-a_{n}$ for large $n$ is called the fundamental difference.

Theorem 5.3. Assume $5 \leq v \equiv 1 \bmod 4$, and let $m$ be the largest integer satisfying $2^{m}<v$. Then the 1 -additive sequence $(4, v)$ is regular with fundamental difference $D=2^{m+3}(v+1)$ and period

$$
N=2^{m+2} \sum_{j=0}^{(v-1) / 2} 2^{-\#(j)} .
$$

In particular, if $5 \leq v=2^{m}+1$, then

$$
N=2^{m+1}+8 \cdot 3^{m-1},
$$

which confirms an observation by Boston (private communication, 1993). Techniques from [Harborth 1977] and [Stolarsky 1977], coupled with Theorem 5.3, can be applied to prove the following result.

Corollary 5.4. Let $\Delta(v)=N(v) / D(v)$ denote the asymptotic density of $(4, v)$, let $\theta=\log 3 / \log 2 \approx$ 1.58496 , and let $c_{0}=\left(1-2^{1 /(\theta-2)}\right)^{\theta-2}$. For all $k \geq 1$, we have

$$
\begin{aligned}
\frac{1}{4}(2 k+1)^{\theta-2} \leq \Delta(4 k+1) & \leq \frac{1}{4} c_{0}(2 k+1)^{\theta-2} \\
& <0.27261(2 k+1)^{\theta-2}, \\
\lim _{k \rightarrow \infty} \Delta(4 k+1) & =0, \\
\liminf _{k \rightarrow \infty}(2 k)^{2-\theta} \Delta(4 k+1) & =\frac{1}{4}, \\
\limsup _{k \rightarrow \infty}(2 k)^{2-\theta} \Delta(4 k+1) & >\frac{1}{7} 3^{\theta-1}>0.27164 .
\end{aligned}
$$

Proof. Let

$$
\Phi(x)=\sum_{j=0}^{x-1} 2^{-\#(j)} \quad \text { for } \quad x \geq 1,
$$

and note that $\Phi(x)=4 x \cdot \Delta(2 x-1)$ for odd $x$. The proof that

$$
1 \leq x^{1-\theta} \Phi(x) \leq c_{0}
$$

is by induction. If (5.1) is true for $1 \leq x \leq 2^{s}$, we use the recursion

$$
\Phi\left(2^{s}+x\right)=\Phi\left(2^{s}\right)+\frac{1}{2} \Phi(x)
$$


to obtain

$$
\left(\frac{3}{2}\right)^{s}+\frac{1}{2} x^{\theta-1} \leq \Phi\left(2^{s}+x\right) \leq\left(\frac{3}{2}\right)^{s}+\frac{c_{0}}{2} x^{\theta-1} .
$$

Define a new function

$$
\varphi(x, c)=\frac{\left(\frac{3}{2}\right)^{s}+\frac{1}{2} c x^{\theta-1}}{\left(2^{s}+x\right)^{\theta-1}}
$$

for $c \geq 1$ and $0 \leq x \leq 2^{s}$. Then one can check by differentiation that $\varphi$ has exactly one extremum, say $x_{0}$, for fixed $c$. Since $\varphi(0, c)=1, \varphi\left(2^{s}, c\right)=$ $\frac{1}{3}(2+c)$ and $\varphi\left(x_{0}, c_{0}\right)=c_{0}$, we deduce that the minimum value of $\varphi(x, 1)$ is 1 and that the maximum value of $\varphi\left(x, c_{0}\right)$ is $c_{0}$. This proves (5.1) for $1 \leq x \leq 2^{s+1}$ and completes the induction.

We also have

$$
\left(2^{s}-1\right)^{1-\theta} \Phi\left(2^{s}-1\right) \rightarrow 1
$$

as $s \rightarrow \infty$ and

$$
\left(\sum_{i=0}^{s} 2^{2 i}\right)^{1-\theta} \Phi\left(\sum_{i=0}^{s} 2^{2 i}\right) \rightarrow \frac{1}{7} 3^{\theta-1}
$$

as $s \rightarrow \infty$.

Improvements can be made in the lower bound for the limit superior, analogous to the treatment in [Harborth 1977].

It's interesting that $\theta$ is the fractal dimension of Pascal's triangle modulo 2 [Wolfram 1984]. This is not surprising in view of the origin of Lemma 3.1.

\section{QUESTIONS}

We have proved above that the 1-additive sequence $(4,4 k+1)$, for $k \geq 1$, has precisely three even terms, and we have determined formulas for the fundamental difference and period as well. Conjecture 4 and part of Conjecture 3 in [Finch 1992b] have therefore been solved.

A resolution of similar issues for $(4,4 k+3)$, for $k \geq 1$, is unlikely soon. The chief reason for this pessimism is that no empirical patterns have been discovered for the fundamental differences [Finch $1992 \mathrm{~b}$ ], so closed-form expressions analogous to the ones in Lemma 3.1 do not seem to exist. A second reason is that these sequences often have extended transient phases prior to the onset of regularity, which makes computations more difficult for the case $(4,4 k+3)$ than for $(4,4 k+1)$. A third reason is that the relevant indicator sequences $\left\{y_{n}\right\}$, given for $n>8 k+8$ by

$y_{n}=\delta\left(y_{n-2}+y_{n-4 k-5}+y_{n-8 k-8}-1\right)$,

$y_{n}=1$ if and only if $2(n-6 k-8)+3 \in\langle 4,4 k+3\rangle$,

with initial conditions

$$
\left(y_{1}, y_{2}, \ldots, y_{8 k+7}, y_{8 k+8}\right)=(0,0, \ldots, 0,1),
$$

do not generally display patterns akin to Pascal's triangle, which, as we recall, is what led to the proof of Lemma 3.1.

There is, however, an exception. The special 1additive sequence $(4,4 k+3)$ with $k=2^{m}-1$, where $m \geq 1$, has the following closed-form expression for $y_{n}$, where $1 \leq n \leq n_{0}=32(k+1)^{2}$. Write $n$ as $n=16(k+1) l+4 c+r$ with $l \geq-1,2 k+2 \leq c \leq$ $6 k+5$ and $1 \leq r \leq 4$. Then $y_{n}$ equals

$\begin{array}{ll}0 & \text { if } r=1 \text { and } 2 k+2 \leq c \leq 3 k+2 ; \\ z(c-3 k-3, l) & \text { if } r=1 \text { and } 3 k+3 \leq c \leq 5 k+4 ; \\ z(c-5 k-5, l+1) & \text { if } r=1 \text { and } 5 k+5 \leq c \leq 5 k+l+5 ; \\ 0 & \text { if } r=1 \text { and } 5 k+l+6 \leq c \leq 6 k+5 ; \\ z(c-2 k-2, l) & \text { if } r=2 \text { and } 2 k+2 \leq c \leq 4 k+3 ; \\ 0 & \text { if } r=2 \text { and } 4 k+4 \leq c \leq 6 k+5 ; \\ 0 & \text { if } r=3 \text { and } 2 k+2 \leq c \leq 5 k+4 ; \\ z(c-5 k-5, l+1) & \text { if } r=3 \text { and } 5 k+5 \leq c \leq 5 k+l+5 ; \\ 0 & \text { if } r=3 \text { and } 5 k+l+6 \leq c \leq 6 k+5 ; \\ 0 & \text { if } r=4 \text { and } 2 k+2 \leq c \leq 2 k+l+1 ; \\ z(c-2 k-1, l) & \text { if } r=4 \text { and } 2 k+l+2 \leq c \leq 4 k+2 ; \\ 0 & \text { if } r=4 \text { and } 4 k+3 \leq c \leq 4 k+l+3 ; \\ z(c-4 k-3, l) & \text { if } r=4 \text { and } 4 k+l+4 \leq c \leq 6 k+4 ; \\ \delta(l+1) & \text { if } r=4 \text { and } c=6 k+5 .\end{array}$

Here we have two kinds of structures: Pascal's triangles as before, and Pascal's triangles cut in half along a diagonal. The upper bound $n_{0}$ on $n$ was chosen so that we might make the following conjecture:

Conjecture 6.1. Suppose that $20 k+23<n<n_{0}$. Then

$$
\sum_{\substack{p+q=n \\ p<q}} y_{p} y_{q} \neq 1 \quad \text { and } \quad \sum_{\substack{p+q=n_{0} \\ p<q}} y_{p} y_{q}=1 .
$$


A corollary of this result would be that the sequence $\left(4,2^{m+2}-1\right)$, for $m \geq 1$, has at least four even terms. We have not attempted a proof of this, although it would be, in principle, similar to Lemma 4.1.

It is known that the 1 -additive sequence $(4,7)$ has fundamental difference $D=11,301,098$ and period $N=1,927,959$ after a transient phase of approximately $1.36 \times 10^{7}$ terms [Finch $1992 \mathrm{~b}$ ]. No periodicity has been detected for the 1-additive sequence $(4,15)$, even up to $2.12 \times 10^{11}$ terms. It is doubtful that significant progress can be made concerning $\left(4,2^{m+2}-1\right)$ soon, given the computational barriers involved.

Some progress has been made on Conjecture 8 of [Finch 1992b]. Assume that the 1-additive sequence $(7, v)$, where $v \geq 8$ is even, has precisely $2+\frac{1}{2} v$ even terms. Cassaigne has developed a computer-assisted proof that the fundamental difference

$$
D(7, v)=112(v+2)
$$

for $v \geq 38$. Similar formulas for $D(u, v)$ could be proved under similar assumptions, for odd $u$ satisfying $9 \leq u \leq 19$ and for suitable large even $v$. The problem of writing an expression for $D(u, v)$ valid for infinitely many $u$ remains open.

\section{REFERENCES}

[Finch 1991] S. R. Finch, "Conjectures about $s$-additive sequences", Fibonacci Quart. 29 (1991), 209-214.
[Finch 1992a] S. R. Finch, "On the regularity of certain 1-additive sequences", J. Combin. Theory A60 (1992), 123-130.

[Finch 1992b] S. R. Finch, "Patterns in 1-additive sequences", Experimental Math. 1 (1992), 57-63.

[Harborth 1977] H. Harborth, "Number of odd binomial coefficients", Proc. Amer. Math. Soc. 62 (1977), 19-22.

[Long 1981] C. T. Long, "Pascal's triangle modulo $p$ ", Fibonacci Quart. 19 (1981), 458-463.

[Queneau 1972] R. Queneau, "Sur les suites s-additives", J. Combin. Theory A12 (1972), 31-71. English summary in Math. Rev. 46, 1741.

[Schmerl and Spiegel 1994] J. H. Schmerl and E. Spiegel, "The regularity of some 1-additive sequences", J. Comb. Theory A66 (1994), 172-175.

[Stolarsky 1977] K. B. Stolarsky, "Power and exponential sums of digital sums related to binomial coefficient parity", SIAM J. Appl. Math. 32 (1977), 717730 .

[Ulam 1964] S. M. Ulam, Problems in Modern Mathematics, Interscience, New York, 1964.

[Wolfram 1984] S. Wolfram, "Geometry of binomial coefficients", Amer. Math. Monthly 91 (1984), 566571.

\section{ELECTRONIC AVAILABILITY}

A complete table of the cases in the proof of Lemma 4.1 is available by anonymous ftp from geom.umn.edu, in directory pub/contrib/expmath/finch.

Julien Cassaigne, LITP, Institut Blaise Pascal, 4 place Jussieu, F-75252 Paris Cedex 05, France (Julien.Cassaigne@litp.ibp.fr)

Steven R. Finch, 6 Foster Street, Wakefield, MA 01880 USA, (sfinch@gnu.ai.mit.edu) 